\title{
Institutional Members
}

\section{Corporate Members}

Health On the Net Foundation (HON)

Geneva - Switzerland

www.healthonnet.org

IBM Corporation

Hawthorne, NY - USA

\section{Academic Members}

\section{Aalborg University}

Aalborg - Denmark

www.v-chi.dk

Academic Medical Center (AMC), University of Amsterdam

Amsterdam - The Netherlands

www.amc.nl/index.cfm?sid=534

Capital Medical University School of Nursing

Beijing, China

sn.ccmu.edu.cn/

Columbia University, Department of Biomedical Informatics

New York, NY - USA

www.dbmi.columbia.edu

DuocUC School of Health

Santiago - Chile

www.duoc.cl

Erasmus University Rotterdam with Department of Medical Informatics: Institute of Health Policy and Management

Rotterdam - The Netherlands

www.erasmusmc.nl/med_informatica/?lang=en

European Campus Rottal-Inn of Deggendort Institute of Technology - Medical and Health Informatics

Deggendorf - Germany

www.th-deg.de/mi-m-en

Georg-August-University Goettingen

Goettingen - Germany

www.med.uni-goettingen.de

Harvard Medical School, Center for Biomedical Informatics

Boston, MA - USA

dbmi.hms.harvard.edu/

Hospital Italiano de Buenos Aires, Department of Health Informatics

Buenos Aires - Argentina

www.hospitalitaliano.org.ar/infomed/
Johns Hopkins University

Laurel MD - USA

dhsi.med.jhmi.edu/

Kuwait University, Faculty of Allied Sciences

Sulaibekhat - Kuwait

www.hsc.edu.kw/fahs/dept/health_information/

Nova Southeastern University

Ft. Lauderdale Florida - USA

osteopathic.nova.edu/msbi

OFFIS e.V. R\&D Division Health

Oldenburg - Germany

www.offis.de

Oregon Health and Science University

Portland, OR - USA

www.ohsu.edu/dmice

Peter L. Reichertz Institute for Medical Informatics

University of Braunschweig, Institute of Technology, and

Hannover Medical School

Braunschweig/Hannover - Germany

www.plri.de

Russian Academy of Medical Sciences

Moscow - Russia

portalramn.ru/

Stanford University, Stanford Medical Informatics

Stanford, CA - USA

www.bmir.stanford.edu

SUNY Downstate Medical Center, College of Health Related Professions

Brooklyn, NY - USA

www.downstate.edu/CHRP/informatics/

The University of Texas Health Science Center at Houston

Houston, Texas - USA

sbmi.uth.edu 
UNC Charlotte, North Carolina - Health Informatics, The Graduate School

Charlotte, NC - USA

www.hi.uncc.edu/

Universidad San Sebastián

Santiago, Chile

www.uss.cl

University of Eastern Finland

Kuopio - Finland

www.uef.fi/uef/english

University of Heidelberg

Heidelberg - Germany

www.med.uni-heidelberg.de/mi

University of New Mexico

Albuquerque, NM - USA

www.hsc.unm.edu/library
University of Seville

Seville - Spain

www.eii.us.es

University of Utah

Salt Lake City, UT - USA

www.med.utah.edu/medinfo

University of Victoria

Victoria, BC - Canada

hinf.uvic.ca

University of Washington

Seattle, WA - USA

www.bhi.washington.edu

Vanderbilt University Medical Center

Nashville, TN - USA

www.mc.vanderbilt.edu/dbmi/ 